\title{
Electronic structure study of local dielectric properties of lanthanoid oxide clusters
}

\section{AUTHOR(S):}

Doi, Kentaro; Mikazuki, Yutaka; Sugino, Shinya; Doi, Tatsuki; Szarek, Pawel; Senami, Masato; Shiraishi, Kenji; ... Chikyo, Toyohiro; Yamada, Keisaku; Tachibana, Akitomo

\section{CITATION:}

Doi, Kentaro ... [et al]. Electronic structure study of local dielectric properties of lanthanoid oxide clusters. Japanese Journal of Applied Physic 2008, 47(1): 205-211

\section{ISSUE DATE:}

2008-01

URL:

http://hdl.handle.net/2433/90862

\section{RIGHT:}

(C) 2008 The Japan Society of Applied Physics; この論文は出版社版であ りません。引用の際には出版社版をご確認ご利用ください。; This is not the published version. Please cite only the published version. 
Please also see, errata,

Ph.D. thesis, Pawel Szarek、

京都大学学術情報リポジトリ（京都大学図書館機構）

Szarek, Pawel,

Theoretical Study of Electronic States of Chemical Bonds.

(化学結合の電子状態に関する理論的研究.)

http://hdl. hand le. net/2433/66212 


\section{Electronic Structure Study of Local Dielectric Properties of Lanthanoid Oxide Clusters}

Kentaro Doi, Yutaka Mikazuki, Shinya Sugino, Tatsuki Doi, Pawel Szarek, Masato Senami, Kenji Shiraishi ${ }^{1}$, Hiroshi IwaI ${ }^{2}$, Naoto Umezawa ${ }^{3}$, Toyohiro Chikyo ${ }^{3}$, Keisaku YAMADA ${ }^{4}$, and Akitomo TACHIBANA*

Department of Micro Engineering, Kyoto University, Kyoto 606-8501, Japan

${ }^{1}$ Graduate School of Pure and Applied Sciences, University of Tsukuba, Tsukuba, 305-8571 Ibaraki, Japan

2 Frontier Collaborative Research Center, Tokyo Institute of Technology, Yokohama 226-8502, Japan

${ }^{3}$ National Institute for Materials Science, Tsukuba, Ibataki, 305-0044, Japan

4 Nanotechnology Research Laboratories, Waseda University, Tokyo 169-0041, Japan

(Received

Density functional calculations are performed for lanthanum-oxide clusters in order to study the local dielectric properties of such clusters using the dielectric constant defined at local points. An increase in coordination number brings about an increase in electron population on the central lanthanum atom, leading to an increase in the local dielectric constant.

KEYWORDS: local dielectric constant, lanthanoid oxides, density functional theory, Rigged QED theory

\footnotetext{
*E-mail address: akitomo@scl.kyoto-u.ac.jp
} 
Significant improvements of electronic devices have been supported by rapid progress in ultralarge-scale integrated (ULSI) circuits. Many studies of complementary metal-oxidesemiconductor (CMOS) gate insulators have been performed in academic and industrial fields of material research. The dielectric breakdown of insulators, however, is a serious problem in reliability of advanced electric devices. Recently, the search for advanced materials superior to $\mathrm{SiO}_{2}$ and the renewal of MOS structures have attracted much attention because the pursuit of device performance, which requires the downsizing of the gate insulators, leads to the loss of device reliability. ${ }^{1}$ These problems require urgent solutions for the generation of new CMOS. Many candidate substitutes for $\mathrm{SiO}_{2}$, that shows desirable dielectric properties has been proposed. Some oxides and silicates, e.g., $\mathrm{CeO}_{2},{ }^{2} \mathrm{Pr}_{2} \mathrm{O}_{3}{ }^{3}$ $\mathrm{Al}_{2} \mathrm{O}_{3},{ }^{4}$ and $\mathrm{Zr}$ and Hf silicates, ${ }^{5-8}$ are known to maintain thermodynamical and chemical stabilities on Si substrates. In recent years, lanthanoid-oxide dielectrics have been considered to be good gate insulators because of their high dielectric constants and wide band gaps. $\mathrm{La}_{2} \mathrm{O}_{3}$, for example, showed a dielectric constant of $27^{9}$ and a band gap of 5.5 eV. ${ }^{10}$ Furthermore, some lanthanoid (Ln) elements, e.g., Ce and Sm, have been reported to work as catalysts in the oxidation of Si substrates. ${ }^{11-14}$

In order to obtain computational results consistent with experimental results of Ln compounds, careful treatment of valence electrons of Ln elements is required for electronic structure calculations. ${ }^{15-17}$ In the calculation of cerium oxide, for example, $\mathrm{Ce}$ 4f in $\mathrm{Ce}_{2} \mathrm{O}_{3}$ is treated as part of the inner core, but that in $\mathrm{CeO}_{2}$ should be treated as a valence electron. ${ }^{15}$ Moreover, the composition of lanthanoid oxide films on Si substrates is very complicated and so are their electronic structures. ${ }^{11-14,18,19}$ Transition layers composed of an oxide layer, an silicate layer, and an interfacial layer are observed between the oxide surface and the substrate. Ln atoms are stable as mixed states in the transition layer, and the complicated structure of the oxide layer procuces a complex electronic structure. ${ }^{11-14}$ The characteristics of lanthanoid oxides are further reviewed in ref. $10{ }^{10}$

In this work, we focus on some oxides, such as $\mathrm{La}_{2} \mathrm{O}_{3}, \mathrm{Ce}_{2} \mathrm{O}_{3}, \mathrm{Gd}_{2} \mathrm{O}_{3}, \mathrm{HfO}_{2}$, and $\mathrm{SiO}_{2}$, and calculate their electronic structures and local dielectric properties using the density- 
the importance of the treatment of the valence electrons of $\mathrm{Gd} .^{20}$ we reported that electrons in the $5 \mathrm{~s}^{2}, 5 \mathrm{p}^{6}, 4 \mathrm{f}^{7}, 6 \mathrm{~s}^{2}$, and $5 \mathrm{~d}^{1}$ states should be treated as valence electrons. We follow this treatment in this work. This is because we want to get more precise and detailed dielectric response of valence electrons than the conventional Clausius-Mossotti (CM) relation. ${ }^{21}$ The analysis is based on the Rigged QED theory described elsewhere. ${ }^{22-28}$

\section{Computational Methods}

\subsection{Electronic structure calculation}

We have performed quantum chemical calculations for several small cluster models of lanthanoid monoxides (i.e., $\mathrm{LaO}, \mathrm{CeO}$, and $\mathrm{GdO}$ ) and several tetrahydroxides, such as $\mathrm{La}(\mathrm{OH})_{4}, \mathrm{Hf}(\mathrm{OH})_{4}$, and $\mathrm{Si}(\mathrm{OH})_{4}$ using the density functional theory ${ }^{29}$ with the Molecular Regional DFT (MRDFT) program package. ${ }^{30}$

The calculations for small clusters can clarify details of the chemical-bonding characteristics of metallic species and $\mathrm{O}$ atoms. However, these calculations are not sufficient to understand the electronic structures of the condensed phase. Therefore, the electronic structure of a large-scale model has also been treated in this work using a combined quantum mechanics / molecular mechanics (QM/MM) method.

Following our previous results, ${ }^{20}$ the electronic configurations of La, Ce, and Gd have been taken as $5 \mathrm{~s}^{2} 5 \mathrm{p}^{6} 5 \mathrm{~d}^{1} 6 \mathrm{~s}^{2}, 4 \mathrm{f}^{1} 5 \mathrm{~s}^{2} 5 \mathrm{p}^{6} 5 \mathrm{~d}^{1} 6 \mathrm{~s}^{2}$, and $4 \mathrm{f}^{7} 5 \mathrm{~s}^{2} 5 \mathrm{p}^{6} 5 \mathrm{~d}^{1} 6 \mathrm{~s}^{2}$ for valence electrons with 46 core electrons, respectively. On the other hand, the 4f, 5s, and 5p states of Hf are located at levels so deep that these states do not contribute to bonding interactions and can be treated as core states. The core electrons are substituted by effective core potentials (ECPs) using the CEP-31G basis set of Stevens et al. ${ }^{31,32}$ Additionally, the f-type polarization function of La is optimized. The exponent of the f function that minimizes the total energy of $\mathrm{LaO}$ is determined to be 0.525 . The contribution of the $\mathrm{f}$ orbital to the interaction between $\mathrm{Ln}$ and $\mathrm{O}$ has also been studied by many researchers, ${ }^{11-17}$ employing the $6-31 \mathrm{G}^{*}$ basis set for $\mathrm{O}$ and $\mathrm{Si}$, and the $6-31 \mathrm{G}^{* *}$ set for $\mathrm{H}$. In DFT calculations, the Lee-Yang-Parr (LYP) ${ }^{33}$ gradient-corrected functionals for correlation interaction are employed, and Becke's hybrid three parameters ${ }^{34}$ for generalized-gradient-approximation 
accurate results for gadolinium oxide clusters in our previous work. ${ }^{20}$

In this work, geometric optimizations and electronic structure calculations for each cluster model are carried out using the GAUSSIAN 03 program package. ${ }^{35}$

\subsection{Electron energy density and local dielectric constant}

Using electron wave functions, local electronic properties such as the electronic stress tensor density and local dielectric constant are calculated. According to the Rigged QED theory, ${ }^{22-28}$ the electronic stress tensor density $\overleftrightarrow{\tau}^{S}(\boldsymbol{r})$ is given by a $3 \times 3$ matrix described as

$$
\begin{array}{r}
\tau^{S k l}(\boldsymbol{r})=\frac{\hbar^{2}}{4 m} \sum_{i} \nu_{i}\left(\psi_{i}^{*}(\boldsymbol{r}) \frac{\partial^{2} \psi_{i}(\boldsymbol{r})}{\partial x^{k} \partial x^{l}}-\frac{\partial \psi_{i}^{*}(\boldsymbol{r})}{\partial x^{k}} \frac{\partial \psi_{i}(\boldsymbol{r})}{\partial x^{l}}\right. \\
\left.+\frac{\partial^{2} \psi_{i}^{*}(\boldsymbol{r})}{\partial x^{k} \partial x^{l}} \psi_{i}(\boldsymbol{r})-\frac{\partial \psi_{i}^{*}(\boldsymbol{r})}{\partial x^{l}} \frac{\partial \psi_{i}(\boldsymbol{r})}{\partial x^{k}}\right),
\end{array}
$$

where $\{k, l\}=\{1,2,3\}, m$ is the electron mass, $\nu_{i}$ is the occupation number of the $i$ th state, and $\psi_{i}(\boldsymbol{r})$ is the electron wave function. ${ }^{27,28}$

The dielectric constant density $\overleftrightarrow{\epsilon}(\boldsymbol{r})$ is locally defined as a $3 \times 3$ matrix described as

$$
\overleftrightarrow{\epsilon}(\boldsymbol{r})=(1-4 \pi \overleftrightarrow{\alpha}(\boldsymbol{r}))^{-1}
$$

where $\stackrel{\leftrightarrow}{\alpha}(\boldsymbol{r})$ is the local polarizability tensor density that satisfies

$$
\boldsymbol{P}(\boldsymbol{r})=\overleftrightarrow{\alpha}(\boldsymbol{r}) \boldsymbol{D}(\boldsymbol{r})
$$

In eq. (2.3), $\boldsymbol{D}(\boldsymbol{r})$ is the electric displacement of the external environment filled with medium M, and $\boldsymbol{P}(\boldsymbol{r})$ is the polarization of system A embedded in medium $\mathrm{M}^{28}$ The local dielectric properties are related to the polarization of the system induced by the electric displacement of the external medium. The polarization $\boldsymbol{P}(\boldsymbol{r})$ is represented as

$$
\begin{aligned}
& \boldsymbol{P}(\boldsymbol{r})=\frac{1}{4 \pi} \operatorname{grad} A_{0_{\mathrm{A}}}(\boldsymbol{r}), \\
& A_{0_{\mathrm{A}}}(\boldsymbol{r})=\int_{\mathrm{A}} d^{3} \boldsymbol{s} \frac{\rho(\boldsymbol{s})}{|\boldsymbol{r}-\boldsymbol{s}|},
\end{aligned}
$$

where $A_{0_{\mathrm{A}}}$ is the gauge potential at $\boldsymbol{r}$, obtained by integrating the charge density in system A. The response of electrons against the external electric field $\boldsymbol{D}(\boldsymbol{r})$ is calculated by the 
out under the condition of homogeneous $\boldsymbol{D}(\boldsymbol{r})$ such as $\boldsymbol{D}(\boldsymbol{r})=$ De composed of the magnitude $D$ and the unit vector $\boldsymbol{e}$. Electronic polarization is calculated using eqs. (2.4) and (2.5), and then the local dielectric constant is derived from eqs. (2.2) and (2.3). As a result of these calculations, the relationship between chemical bonding properties and local dielectric constants are determied because these quantities are derived from the same electron wave functions. ${ }^{28}$

The electronic stress tensor density and the local dielectric constant are calculated using the MRDFT program package. ${ }^{30}$

\section{Results and Discussion}

\subsection{Electronic structures of $\mathrm{LnO}$}

The optimized bond lengths of $\mathrm{LaO}, \mathrm{CeO}$, and $\mathrm{GdO}$ are shown in Table I. The calculated bond lengths of $\mathrm{CeO}$ and $\mathrm{GdO}$ show good agreement with the experimental data. ${ }^{36,37}$ The calculated bond length of $\mathrm{LaO}$ was improved with the basis set that contains the ftype polarization function. According to the increase in atomic number, the radius of lanthanoid atoms thnds to decrease because the addition of electrons to the $4 \mathrm{f}$ orbitals does not shield the atomic nucleus. This is known as lanthanoid contraction. In the case of the lanthanum oxide, the unoccupied $\mathrm{f}$ function is considered in the electronic structure calculations as the polarization function, and the overlap between $\mathrm{La}(4 \mathrm{f})$ and $\mathrm{O}(2 \mathrm{p})$ stabilizes the chemical bond. Thus, hereafter, the electronic structures of lanthanum oxide are obtained using the CEP-31G plus f-type polarization function.

The MO interaction diagrams of $\mathrm{LaO}, \mathrm{CeO}$, and $\mathrm{GdO}$ are shown in Fig. 1. The 5p and $5 \mathrm{~d}$ orbitals are delocalized and contribute to the interaction between Ln and O. On the other hand, the $4 \mathrm{f}$ and $5 \mathrm{~s}$ orbitals remain intact in the Ln atom. In the case of LaO, the MO levels are divided into two bands, with each band showing a different property. In the lower-energy band, the interaction between La 5p and $\mathrm{O} 2 \mathrm{~s}$ generates bonding and anti bonding MOs because of the close energy levels of these two atomic orbitals (AOs), $-0.881(-23.968)$ and -0.897 a.u. (-24.419 eV), respectively, and of their non zero overlap interaction. In the higher-energy band, MO levels composed of La 5p,5d 
interaction of $\mathrm{La} 5 \mathrm{p}-\mathrm{O} 2 \mathrm{p}$. In the cases of $\mathrm{CeO}$ and $\mathrm{GdO}$, the orbital interactions are explained as in the case of $\mathrm{LaO}$.

\subsection{Electronic structures of $\mathrm{La}(\mathrm{OH})_{4}, \mathrm{Hf}(\mathrm{OH})_{4}$, and $\mathrm{Si}(\mathrm{OH})_{4}$}

Next, the electronic structures of $\mathrm{La}(\mathrm{OH})_{4}, \mathrm{Hf}(\mathrm{OH})_{4}$, and $\mathrm{Si}(\mathrm{OH})_{4}$ are calculated in order to analyze the bonding states between metallic species and $\mathrm{O}$ atoms in oxygen ligand systems. This discussion is motivated by the interest for the many folded Ln-O interactions. In the calculations, all molecules were fixed in tetragonal symmetry $\left(T_{d}\right)$. In the ground state, the bond lengths of $\mathrm{La}-\mathrm{O}, \mathrm{Hf}-\mathrm{O}$, and $\mathrm{Si}-\mathrm{O}$ were optimized at 2.24, 1.92, and $1.60 \AA$, respectively. The angles $\angle \mathrm{M}-\mathrm{O}-\mathrm{H}(\mathrm{M}=\mathrm{La}$, Hf, and $\mathrm{Si})$ were fixed at $180^{\circ}$. Figure 2 shows MO interaction diagrams of $\mathrm{La}(\mathrm{OH})_{4}, \mathrm{Hf}(\mathrm{OH})_{4}$, and $\mathrm{Si}(\mathrm{OH})_{4}$. In the cases of $\mathrm{La}(\mathrm{OH})_{4}$, La $6 \mathrm{~s}$ and $\mathrm{O} 2 \mathrm{~s}, 2 \mathrm{p}$ interact and generate a bonding orbital. The singly occupied molecular orbital (SOMO) is a non bonding $\mathrm{O} 2 \mathrm{p}$ orbital. The bonding state of La-O, however, shows almost the same characteristics as those of LaO.

It is interesting to understand the similarities and differences between $\mathrm{La}_{2} \mathrm{O}_{3}$ and $\mathrm{HfO}_{2}$ because $\mathrm{HfO}_{2}$ is known as a good candidate gate insulator, so that $\mathrm{HfO}_{2}$ has reference properties as an advanced insulator. In $\mathrm{HfO}_{2}$, the interactions between $\mathrm{Hf} 5 \mathrm{p}$ and $\mathrm{O} 2 \mathrm{~s}, 2 \mathrm{p}$ are considered to be weak because the calculated Hf 5p level of -1.408 a.u. $(-38.327 \mathrm{eV})$ is deeper than $\mathrm{O} 2 \mathrm{~s}$ at -0.897 a.u. $(-24.419 \mathrm{eV})$. The differences in bonding properties between La-O and Hf- $\mathrm{O}$ clearly shown in Fig. 2. In $\mathrm{Hf}(\mathrm{OH})_{4}$, the $\mathrm{O} 2 \mathrm{~s}$ level is weakly influenced by Hf $5 \mathrm{p}$. The bonding orbital generated from $\mathrm{Hf} 5 \mathrm{~d}-\mathrm{O} 2 \mathrm{p}$ is more stable than that generated from La $5 \mathrm{~d}-\mathrm{O} 2 \mathrm{p}$ in $\mathrm{La}(\mathrm{OH})_{4}$ because of the weak contribution of the anti bonding element caused by the Hf 5 p orbital. On the other hand, in the case of $\mathrm{Si}(\mathrm{OH})_{4}$, the bond between $\mathrm{Si}$ and $\mathrm{O}$ is caused by the interactions of $\mathrm{Si} 3 \mathrm{~s}-\mathrm{O} 2 \mathrm{~s}$ and $\mathrm{Si} 3 \mathrm{~s}, 3 \mathrm{p}-\mathrm{O} 2 \mathrm{p}$, as observed in the $\mathrm{SiO}_{2}$ bulk system.

\subsection{Stress tensor density}

The stress tensor density represented by eq. (2.1) explains local electronic stresses and bonding characteristics. Figures 3(a) and 3(b) show maps of the stress tensor density for $\mathrm{La}(\mathrm{OH})_{4}$ and $\mathrm{Hf}(\mathrm{OH})_{4}$, respectively. In these figures, one $\mathrm{Hf}$ or La atom and two $\mathrm{O}$ atoms 

of the diagonalized stress tensor density are plotted at each point. The positive or negative quantities around the La, Hf, and O atoms explain the tensile and compressive stresses of the electron density, respectively. The tensile stress observed between the La or Hf and O atoms connected to each other is called the "spindle structure." 27 The stress tensor density is calculated from the derivatives of the electron wave functions, and the chemical bonding properties contained in the wave functions are clarified on the basis of the stress tensor density as the dimension of energy density. In particular, in covalent bonds, electron density experiences tensile stress because of the attractive interactions between atoms. In this work, only the third principal axis, which corresponds to the largest eigenvalue, is only focused on because the characteristics of chemical bonds are clarified best on this axis. More details and some examples of the stress tensor density are shown elsewhere. ${ }^{27}$ The difference shown in $\mathrm{Hf}-\mathrm{O}$ and $\mathrm{La}-\mathrm{O}$ is caused by the interaction between La or $\mathrm{Hf}$ and $\mathrm{O}$ atoms, as described above using MO interaction diagrams. The electron density between $\mathrm{Hf}$ and $\mathrm{O}$ atoms in $\mathrm{Hf}(\mathrm{OH})_{4}$ experiences more compressive stress than that between La and $\mathrm{O}$ in $\mathrm{La}(\mathrm{OH})_{4}$. This is because the chemical bond Hf-O is mainly composed of bonding orbitals formed by $\mathrm{Hf} 5 \mathrm{~d}-\mathrm{O} 2 \mathrm{p}$ and $\mathrm{Hf} 6 \mathrm{~s}-\mathrm{O} 2 \mathrm{p}$, and in this case, the contribution of $\mathrm{Hf}$ $5 \mathrm{p}$ is very weak. On the other hand, in $\mathrm{La}(\mathrm{OH})_{4}$, La $5 \mathrm{p}$ interacts with $\mathrm{O} 2 \mathrm{~s}, 2 \mathrm{p}$ and causes anti bonding components. Therefore, the electron density that contributes to the Hf-O bond shows a more compressive property. The MO diagram is spatially visualized using the stress tensor density. In $\mathrm{Si}(\mathrm{OH})_{4}$, as shown in Fig. 3(c), the electron density between $\mathrm{Si}$ and $\mathrm{O}$ atoms experiences tensile stress in the regions represented by positive quantities. The bonding character of $\mathrm{Si}-\mathrm{O}$ in $\mathrm{Si}(\mathrm{OH})_{4}$ shows different images from those of $\mathrm{La}(\mathrm{OH})_{4}$ and $\mathrm{Hf}(\mathrm{OH})_{4}$. One of the reasons for this difference is the difference in the number of electrons that contribute to the bonds. Mulliken atomic charges ${ }^{38}$ in these molecules are shown in Table II. As a result, the charge transfer between metallic species and an $\mathrm{O}$ atom is the greatest in $\mathrm{Hf}(\mathrm{OH})_{4}$ and least in $\mathrm{Si}(\mathrm{OH})_{4}$, so that electrons are exchanged most prominently between $\mathrm{Hf}$ and $\mathrm{O}$ atoms and that the chemical bonds between them become stronger. 
The local properties of dielectric constants are calculated using eqs. (2.2)-(2.5) and the results are shown in Fig. 4. The local dielectric constant depends on the electronic polarization $\boldsymbol{P}(\boldsymbol{r})$ defined by eq. (2.3), and the responses of such polarization to the external electric fields $\boldsymbol{D}(\boldsymbol{r})$ are attributed to the polarizability $\stackrel{\leftrightarrow}{\alpha}(\boldsymbol{r})$. Therefore, the local dielectric tensor represented by eq. (2.2) is calculated from the polarizability at each point. In this work, polarizability was calculated from numerical finite differences. The response of electrons to the external electric field calculated using the Schrödinger equation shows quantum mechanical perspectives. The electron density derived from the Schrödinger equation is drawn using continuous wave functions and not discrete values, so that the dielectric constant that depends on electronic polarization is defined at each point in a function space. The absolute largest eigenvalues and eigenvectors of the diagonalized dielectric constants are shown on the same cross sections in Fig. 3. In this discussion, the largest dielectric constants are only focused on because the distribution of the largest eigenvalues clearly describes the relationship between electrodynamics and chemical bonding properties.

The distributions of the dielectric constants clearly differ between $\mathrm{La}(\mathrm{OH})_{4}, \mathrm{Hf}(\mathrm{OH})_{4}$, and $\mathrm{Si}(\mathrm{OH})_{4}$. High dielectric constants were obtained between $\mathrm{Hf}$ and $\mathrm{O}$ atoms in $\mathrm{Hf}(\mathrm{OH})_{4}$, as shown in Fig. 4(b). On the other hand, no such result was obtained in $\mathrm{La}(\mathrm{OH})_{4}$ [Fig. 4(a)]. In the case of $\mathrm{Si}(\mathrm{OH})_{4}$, as shown in Fig. 4(c), large values were observed between $\mathrm{O}$ atoms, not between $\mathrm{Si}$ and $\mathrm{O}$ atoms. The dielectric constant originates from the response of electrons to the external electric field $\boldsymbol{D}(\boldsymbol{r})$, so that the inner electric field $\boldsymbol{E}(\boldsymbol{r})$ changes as a function of $\boldsymbol{D}(\boldsymbol{r})$. In the regions where the dielectric constant is larger than 1.0, it is concluded that polarization caused by electron displacements is easily induced by $\boldsymbol{D}(\boldsymbol{r})$. In $\mathrm{Hf}(\mathrm{OH})_{4}$, in particular, electrons that contribute to the bond between $\mathrm{Hf}$ and $\mathrm{O}$ atoms responded to $\boldsymbol{D}(\boldsymbol{r})$, generated polarization, and caused a high dielectric constant.

Furthermore, Fig. 4(d) shows the result of an anionic state $\mathrm{La}(\mathrm{OH})_{4}^{-}$. In an ideal ionic crystal of $\mathrm{La}_{2} \mathrm{O}_{3}$, La and $\mathrm{O}$ are considered to be ionized as $\mathrm{La}^{3+}$ and $\mathrm{O}^{2-}$, respectively. Therefore, anionic lanthanum tetrahydroxide, $\mathrm{LaO}_{4} \mathrm{H}_{4}^{-}$, was calculated in order to realize 
optimized at $2.26 \AA$. The distribution of the dielectric constants of $\mathrm{La}(\mathrm{OH})_{4}^{-}$showed the same tendency as that of $\mathrm{Hf}(\mathrm{OH})_{4}$. An added electron responded to $\boldsymbol{D}(\boldsymbol{r})$ and the polarization appeared around a center La atom. As shown in Table II, the calculated Mulliken atomic charges of La and $\mathrm{O}$ atoms in $\mathrm{La}(\mathrm{OH})_{4}^{-}$are 1.164 and -0.793 , respectively, which proves that the added electron occupied atomic orbitals of La and $\mathrm{O}$ and contributed to the La-O bond similarly to the $\mathrm{Hf}-\mathrm{O}$ bond in $\mathrm{Hf}(\mathrm{OH})_{4}$.

In order to confirm the results presented above, the local dielectric constant was calculated for a large-cluster model of lanthanum silicate. The electronic structure of this large cluster was calculated using the QM/MM method. In the QM region, electron wave functions were expanded by the same basis set as those in $\mathrm{LaO}$ and $\mathrm{La}(\mathrm{OH})_{4}$. However, in the MM region, atomic force fields were represented by the universal force field (UFF). The silicate model was composed of $234 \mathrm{Si}$ and $394 \mathrm{O}$ atoms in the MM region and $7 \mathrm{Si}$ and $8 \mathrm{O}$ atoms in the $\mathrm{QM}$ region. As a result, in the optimized structure, the coordination number was 8, and $8 \mathrm{O}$ atoms existed within $3.0 \AA$ from a La atom. Several cross sections in which one La atom and two $\mathrm{O}$ atoms were located showed the distribution of the dielectric constant in the disordered condensed phase. As shown in Fig. 5, high dielectric constants were observed between La and $\mathrm{O}$ atoms. These distributions explain a tendency similar to that in the anionic state such as $\mathrm{La}(\mathrm{OH})_{4}^{-}$, so that charges from the environment are accepted in the interactions between La and $\mathrm{O}$ atoms, thereby causing high dielectric constants.

It is concluded that La atoms in silicate or interfacial layers where charge transfer is easily result in high dielectric constants, which neutral 4-coordinated lanthanum oxides do not show.

\section{Conclusion}

In this work, we investigated the local properties of dielectric constants, focusing on La, Hf, and Si oxides. Local dielectric constant is defined as a field quantity. The differences in bonding characteristics between $\mathrm{La}-\mathrm{O}, \mathrm{Hf}-\mathrm{O}$, and $\mathrm{Si}-\mathrm{O}$ were visualized using the stress tensor density, and the origin of high dielectric constants was revealed. Electrons that 
electric field, so that no high dielectric constant was observed in $\mathrm{La}(\mathrm{OH})_{4}$. The local properties of a lanthanum silicate were investigated using a QM/MM method, in which charge transfer from the environment to La-O bonds resulted in high dielectric constants, as observed in $\mathrm{La}(\mathrm{OH})_{4}^{-}$and $\mathrm{Hf}(\mathrm{OH})_{4}$.

In our future work, the dielectric constant will be calculated taking into account the molecular vibrational effect. Ionic polarizations will also be calculated to obtain accurate dielectric properties.

\section{Acknowledgements}

A part of the computations was supported by Center for Computational Science, Okazaki, Japan. This work is supported by Special Coordination Funds for Promoting Science and Technology from the Ministry of Education, Culture, Sports, Science and Technology, Japan (MEXT), for which the authors express their gratitude. This work is supported in part by the Center of Excellence for Research and Education on Complex Functional Mechanical Systems (COE Program of MEXT). 
1) J. H. Stathis and D. J. Dimaria: IEDM Tech. Dig., 1998, p. 167.

2) M. Yoshimoto, T. Maeda, T. Ohnishi, G. H. Lee, and H. Louinuma: Mater. Res. Soc. Symp. Proc. 41 (1996) 210.

3) J. P. Liu, P. Zaumseil, E. Bugiel, and H. J. Osten: Appl. Phys. Lett. 79 (2001) 671.

4) E. P. Gusev, M. Copel, E. Cartier, I. J. R. Baumvol, C. Krug, and M. A. Cribelyuk: Appl. Phys. Lett. 76 (2000) 176.

5) G. D. Wilk, R. M. Wallace, and L. M. Anthony: J. Appl. Phys. 89 (2001) 5243.

6) G. D. Wilk and R. M. Wallace: Appl. Phys. Lett. 74 (1999) 2854.

7) G. D. Wilk, R. M. Wallace, and J. M. Anthony: J. Appl. Phys. 87 (2000) 484.

8) G. D. Wilk and R. M. Wallace: Appl. Phys. Lett. 76 (2000) 112.

9) Y. Kim, S. Ohmi, K. Tsutsui, and H. Iwai: in Proc. 34th European Solid-State Device Research Conf., (ESSDERC2004), Leuven, 2004, p. 81.

10) G. Adachi and N. Imanaka: Chem. Rev. 98 (1998) 1479.

11) F. U. Hillebrecht, M. Ronay, D. Rieger, and F. J. Himpsel: Phys. Rev. B 34 (1986) 5377.

12) S. Chang, P. Philip, A. Wall, A. Raisanen, N. Toullier, and A. Franciosi: Phys. Rev. B 35 (1987) 3013.

13) J. Onsgaard, J. Ghijsen, R. L. Johnson, M. Christiansen, F. Ørskov, and P. J. Godowiski: Phys. Rev. B 43 (1991) 4216.

14) P. Patsalas, S. Logothetidis, L. Sygellou, and S. Kennou: Phys. Rev. B 68 (2003) 035104.

15) N. V. Skorodumova, R. Ahuja, S. I. Simak, I. A. Abrikosov, B. Johansson, and B. I. Lundqvist: Phys. Rev. B 64 (2001) 115108.

16) L. Marsella and V. Fiorentini: Phys. Rev. B 69 (2004) 172103.

17) L. Petit, A. Svane, Z. Szotek, and W. M. Temmerman: Phys. Rev. B 72 (2005) 205118.

18) T. Hattori, T. Yoshida, T. Shiraishi, K. Takahashi, H. Nohira, S. Joumori, K. Nakajima, M. Suzuki, K. Kimura, I. Kashiwagi, C. Ohshima, S. Ohmi, and H. Iwai: 
19) H. Nohira, T. Shiraishi, K. Takahashi, T. Hattori, I. Kashiwagi, C. Ohshima, S. Ohmi, H. Iwai, S. Joumori, K. Nakajima, M. Suzuki, K. Kimura: Appl. Surf. Sci. 234 (2004) 493.

20) K. Doi, K. Fujitani, N. Kadowaki, K. Nakamura, and A. Tachibana: Jpn. J. Appl. Phys. 44 (2005) 6115.

21) K. Nakamura, K. Doi, K. Fujitani, and A. Tachibana: Phys. Rev. B 71 (2005) 045332.

22) A. Tachibana: Theor. Chem. Acc. 102 (1999) 188.

23) A. Tachibana: J. Chem. Phys. 115 (2001) 3497.

24) A. Tachibana: in Stress Induced Phenomena in Metallization, ed. S. P. Baker (American Institute of Physics, New York, 2002) p. 105.

25) A. Tachibana: in Reviews of Modern Quantum Chemistry; A Celebration of the Contributions of Robert G. Parr, ed. K. D. Sen (World Scientific, Singapore, 2002) Vol. 2, Chap. 45, p. 1327.

26) A. Tachibana: in Fundamental World of Quantum Chemistry, A Tribute to the Memory of Per-Olov Löwdin, eds. E. J. Brändas and E. S. Kryachko (Kluwer Academic, Dordrecht, 2003) Vol. 2, p. 211.

27) A. Tachibana: Int. J. Quantum Chem. 100 (2004) 981.

28) A. Tachibana: J. Mol. Model. 11 (2005) 301.

29) R. G. Parr and W. Yang: Density-Functional Theory of Atoms and Molecules (Oxford University Press, New York, 1989).

30) K. Nakamura, K. Doi and A. Tachibana: Molecular Regional DFT program package, ver. 1, Tachibana Lab., Kyoto University, Kyoto, 2004.

31) W. J. Stevens, M. Krauss, H. Bausch, and P. G. Jasien: Can. J. Chem. 70 (1992) 612.

32) T. R. Cundari and W. J. Stevens: J. Chem. Phys. 98 (1993) 5555.

33) C. Lee, W. Yang, and R. G. Parr: Phys. Rev. B 37 (1988) 785.

34) A. D. Becke: J. Chem. Phys. 98 (1993) 5648.

35) M. J. Frisch et al., Gaussian 03, Revision B.05, Gaussian, Inc., Pittsburgh, PA, 2003.

36) K. P. Huber and G. Herzberg: Molecular Spectra and Molecular Structure; Constants 
37) B. R. Yadav, S. B. Rai, and D. K. Rai: J. Mol. Spectrosc. 89 (1981) 1.

38) R. S. Mulliken: J. Chem. Phys. 23 (1955) 1833. 
Fig. 1. MO interaction diagrams of (a) LaO, (b) CeO, (c) GdO at B3LYP/(CEP-31G, 6-31G*) level. Closed circles represent the occupied levels and dashed lines indicate atomic-orbital contributions to the MOs. Values are written in atomic unit.

Fig. 2. MO interaction diagrams of (a) $\mathrm{La}(\mathrm{OH})_{4}$, (b) $\mathrm{Hf}(\mathrm{OH})_{4}$, and (c) $\mathrm{Si}(\mathrm{OH})_{4}$. Values are written in atomic unit.

Fig. 3. Maps of the electronic stress tensor density for (a) $\mathrm{La}(\mathrm{OH})_{4}$, (b) $\mathrm{Hf}(\mathrm{OH})_{4}$, and (c) $\mathrm{Si}(\mathrm{OH})_{4}$. One metallic atom (La, Hf, or $\mathrm{Si}$ ) and two $\mathrm{O}$ atoms are located on the cross sections. The short lines denote the eigenvectors of the third principal axis corresponding to the largest eigenvalue of the stress tensor density. Contours denote the values of $-0.05,-0.01,0.0,0.01$, and 0.05 .

Fig. 4. Maps of the dielectric constant for (a) $\mathrm{La}(\mathrm{OH})_{4}$, (b) $\mathrm{Hf}(\mathrm{OH})_{4}$, (c) $\mathrm{Si}(\mathrm{OH})_{4}$, and (d) $\mathrm{La}(\mathrm{OH})_{4}^{-}$. The cross sections are the same as those in Fig. 3. The short lines denote the eigenvectors of the third principal axis corresponding to the largest absolute eigenvalue of the dielectric tensor. Contours denote the values of 1.0, 2.0, and 5.0, and areas smaller than 1.0 are shown in white.

Fig. 5. Maps of the dielectric constant for large-cluster model of lanthanum silicate. There are eight $\mathrm{O}$ atoms within $3.0 \AA$ from a La atom. One La atom and two O atoms are located on the cross sections. 
Table I. Bond lengths of lanthanoid monoxides: $\mathrm{LaO}$ with $\mathrm{f}$ and $\mathrm{LaO}$ without $\mathrm{f}$ mean the calculations performed with and without f-type polarization function, respectively. The calculations were performed with a CEP-31G basis set and a LanL2DZ basis set.

\begin{tabular}{|c|c|c|c|c|}
\hline & \multicolumn{4}{|c|}{ Bond length $(\AA)$} \\
\hline & LaO without $\mathrm{f}$ & $\mathrm{LaO}$ with $\mathrm{f}$ & $\mathrm{CeO}$ & $\mathrm{GdO}$ \\
\hline CEP-31G & 1.947 & 1.903 & 1.828 & 1.844 \\
\hline LanL2DZ & 1.965 & 1.914 & - & - \\
\hline Exp. & \multicolumn{2}{|c|}{1.826} & 1.820 & 1.812 \\
\hline
\end{tabular}

Table II. Mulliken atomic charges in $\mathrm{La}(\mathrm{OH})_{4}, \mathrm{La}(\mathrm{OH})_{4}^{-}, \mathrm{Hf}(\mathrm{OH})_{4}$, and $\mathrm{Si}(\mathrm{OH})_{4}$.

\begin{tabular}{lcccc}
\hline & $\mathrm{La}(\mathrm{OH})_{4}$ & $\mathrm{La}(\mathrm{OH})_{4}^{-}$ & $\mathrm{Hf}(\mathrm{OH})_{4}$ & $\mathrm{Si}(\mathrm{OH})_{4}$ \\
\hline $\mathrm{M}(=\mathrm{La}, \mathrm{Hf}$, or $\mathrm{Si})$ & 1.467 & 1.164 & 1.721 & 1.099 \\
$\mathrm{O}$ & -0.683 & -0.797 & -0.767 & -0.599 \\
\hline
\end{tabular}




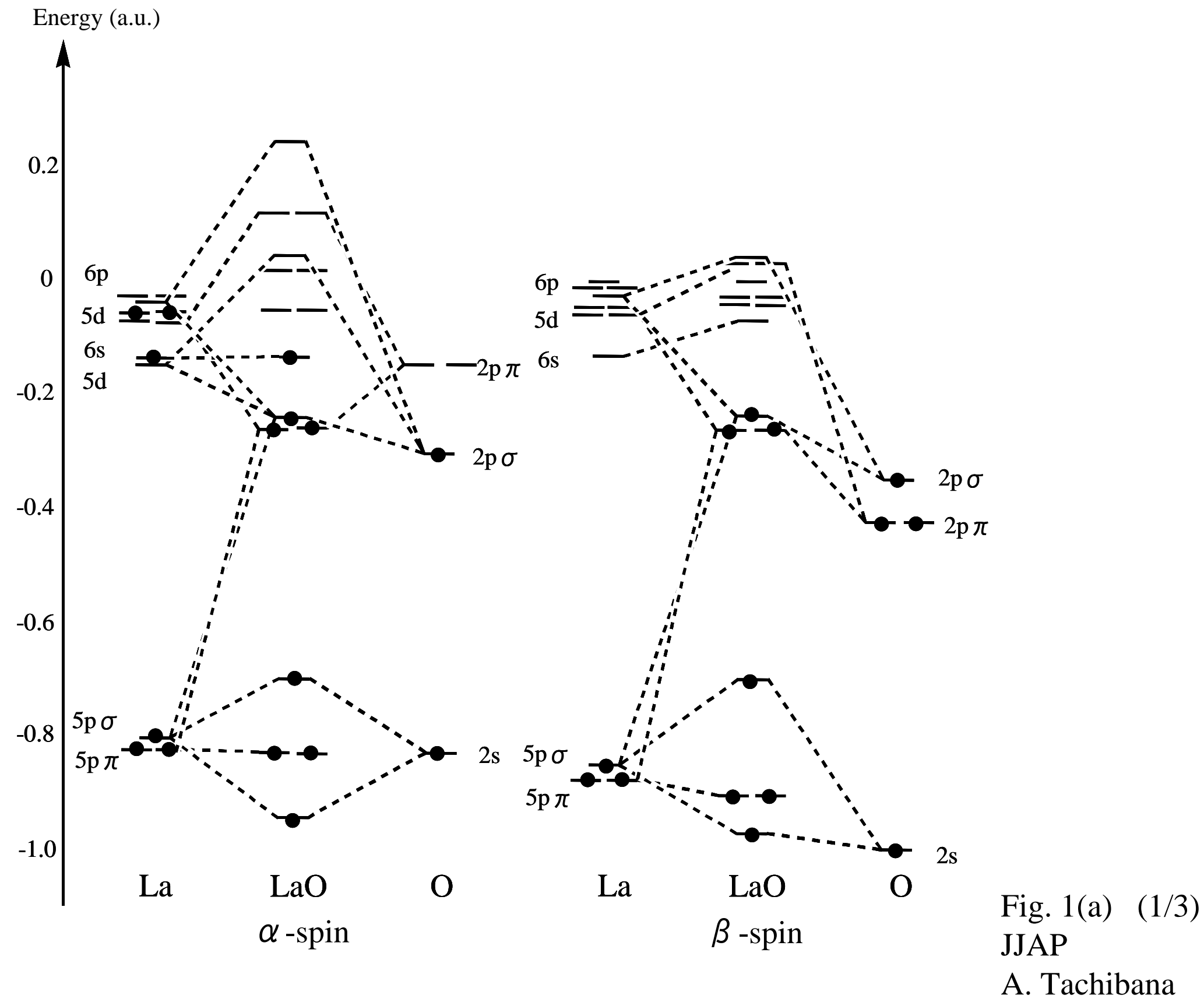



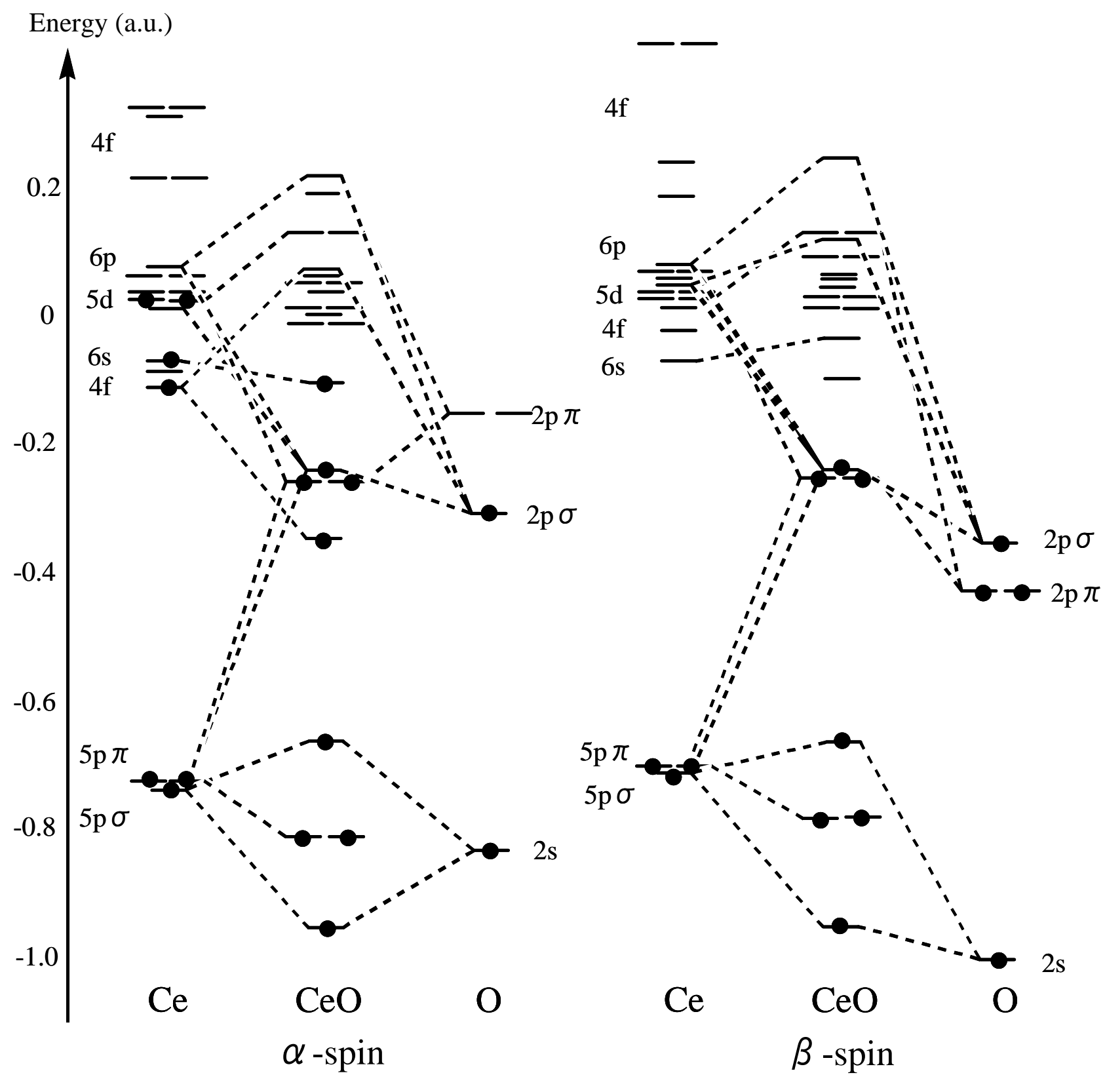

Fig. 1(b) (2/3)

A. Tachibana 

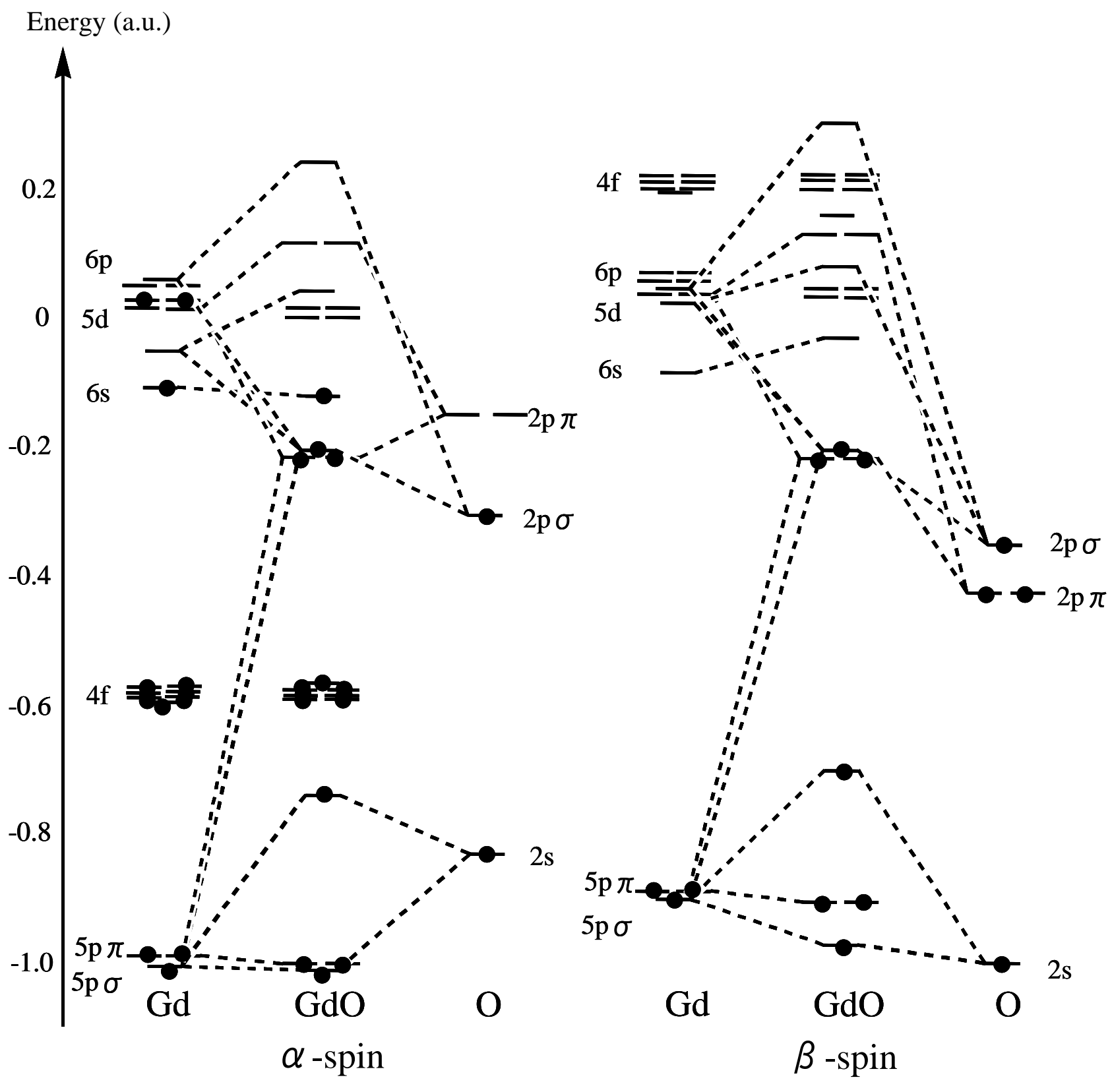

Fig. 1(c) (3/3) JJAP

A. Tachibana 


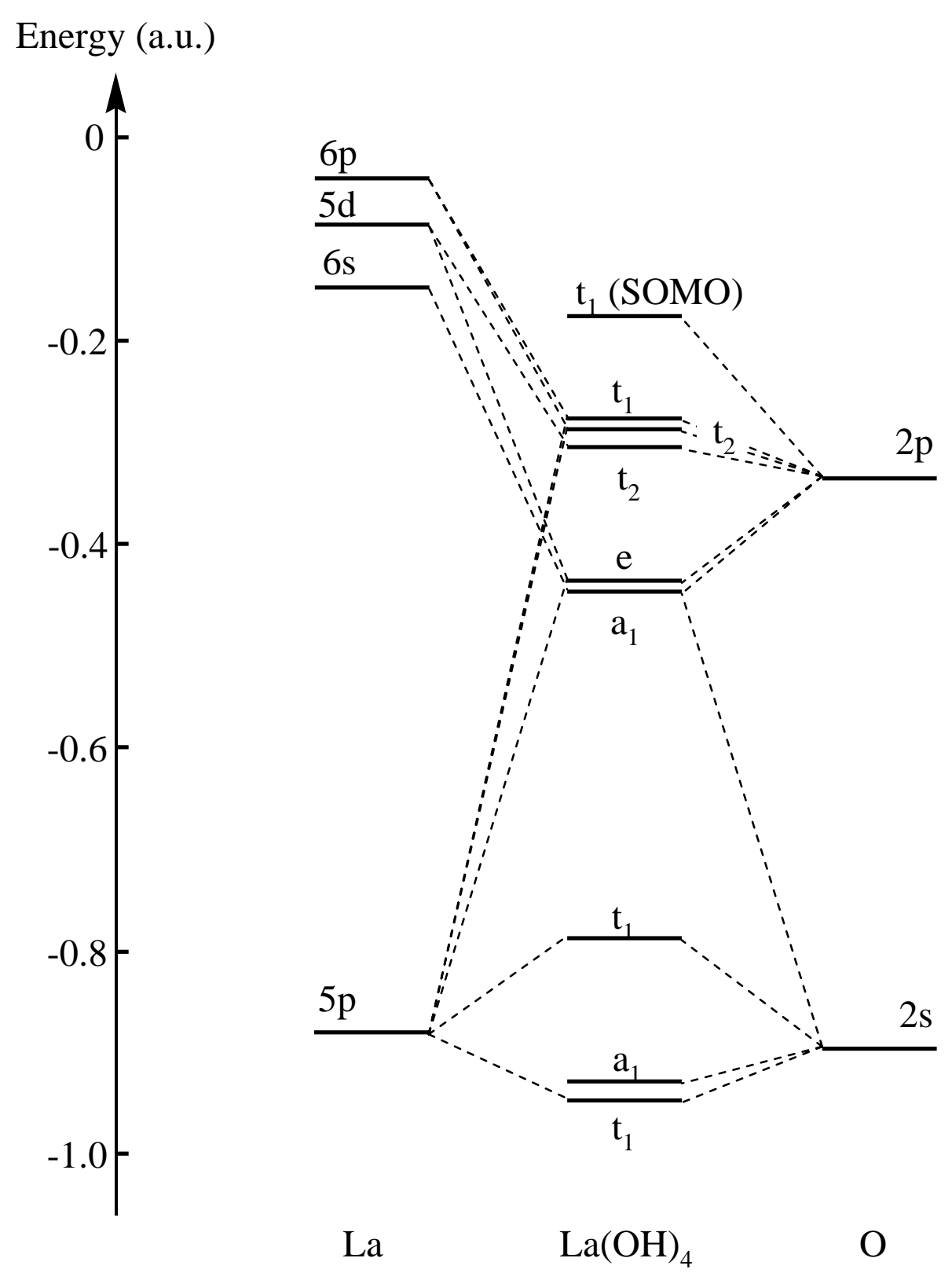

Fig. 2(a) (1/3) JJAP

A. Tachibana 


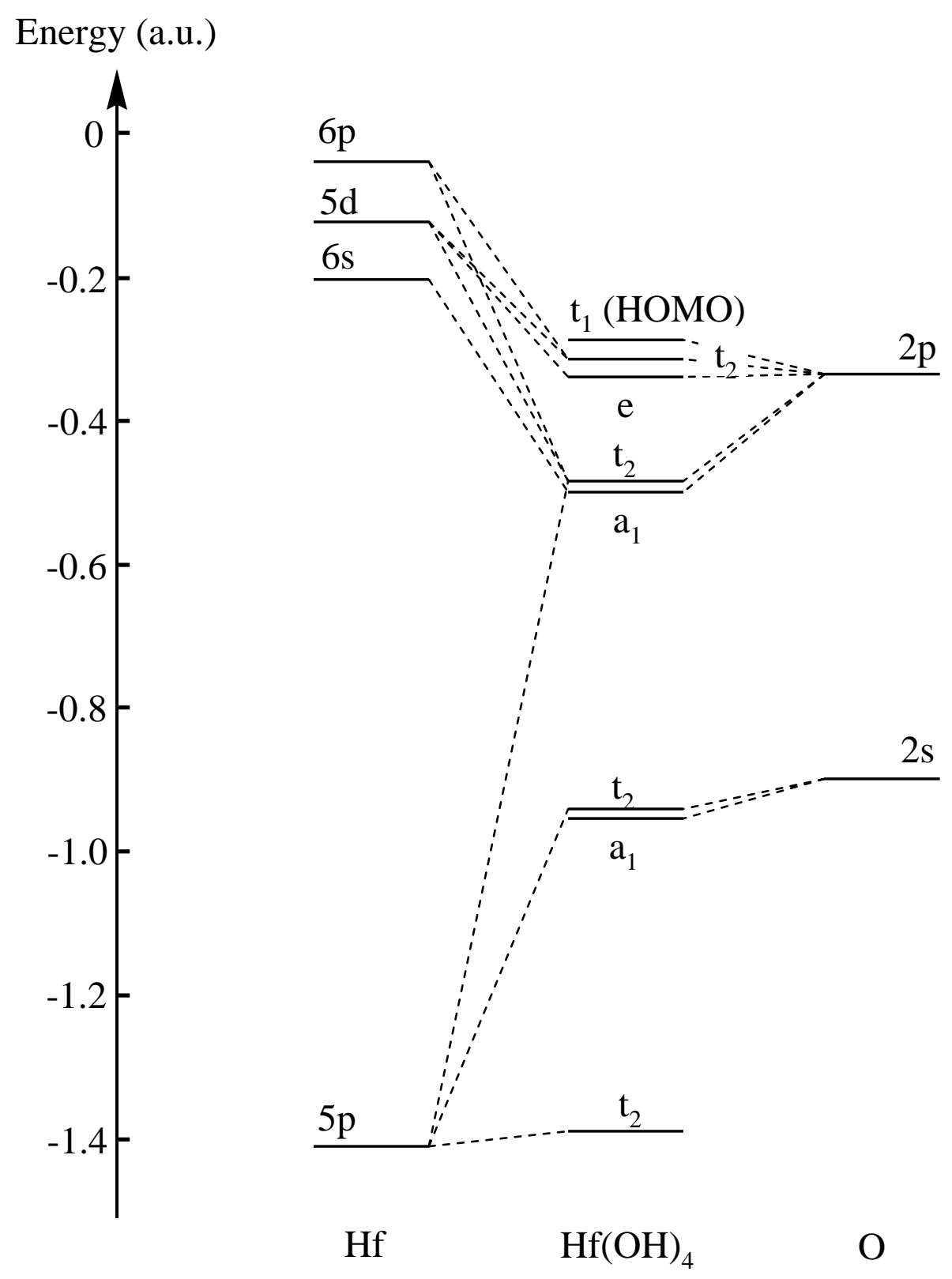

Fig. 2(b) (2/3) JJAP

A. Tachibana 


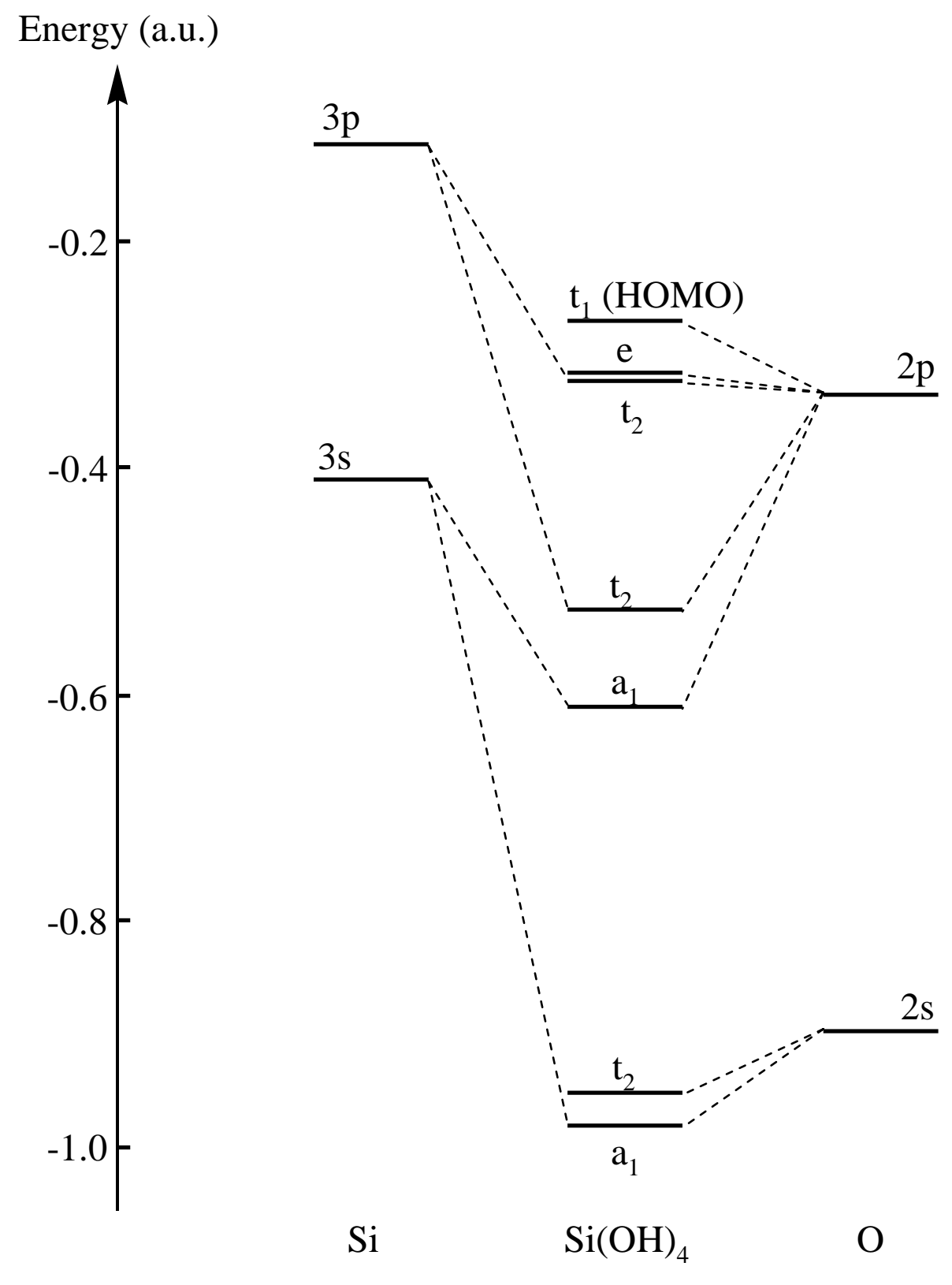

Fig. 2(c) (3/3) JJAP

A. Tachibana 


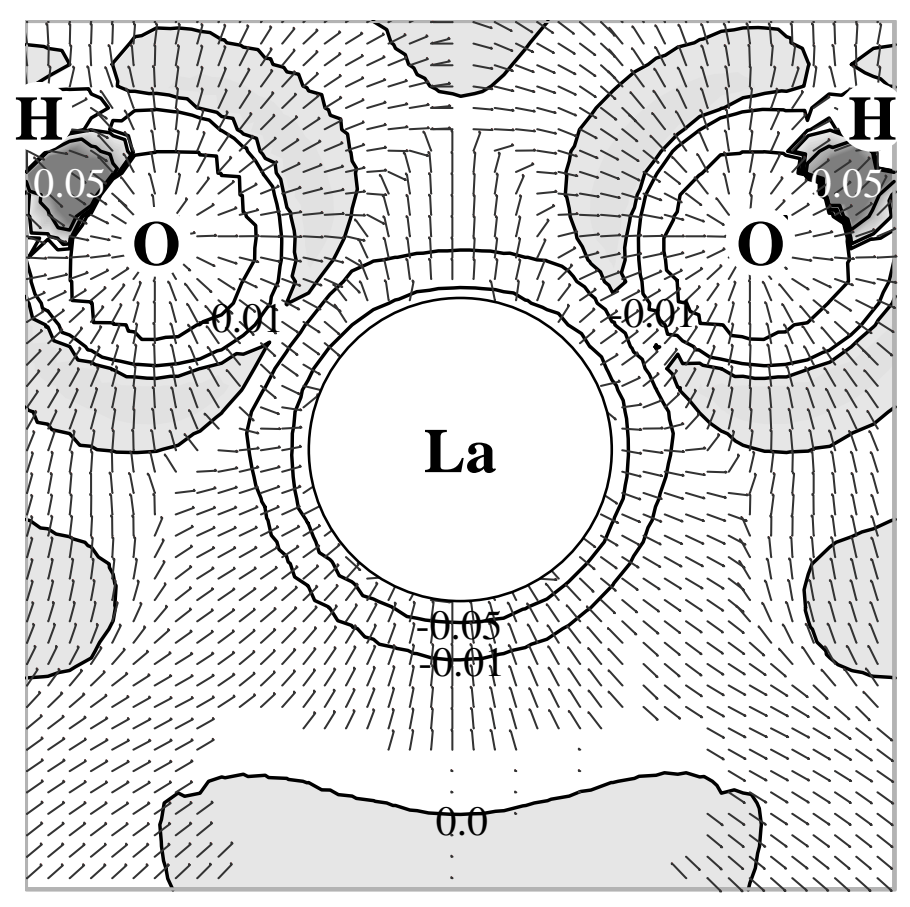

Fig. 3(a) (1/3) JJAP

A. Tachibana 


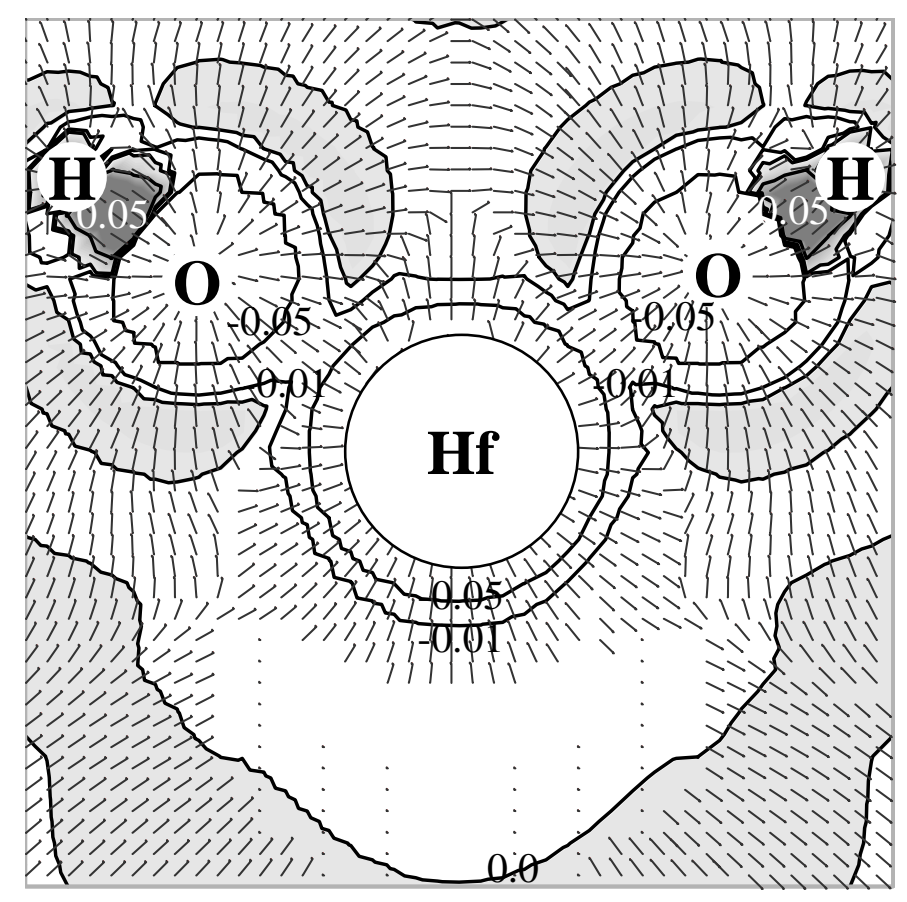

Fig. 3(b) (2/3) JJAP

A. Tachibana 


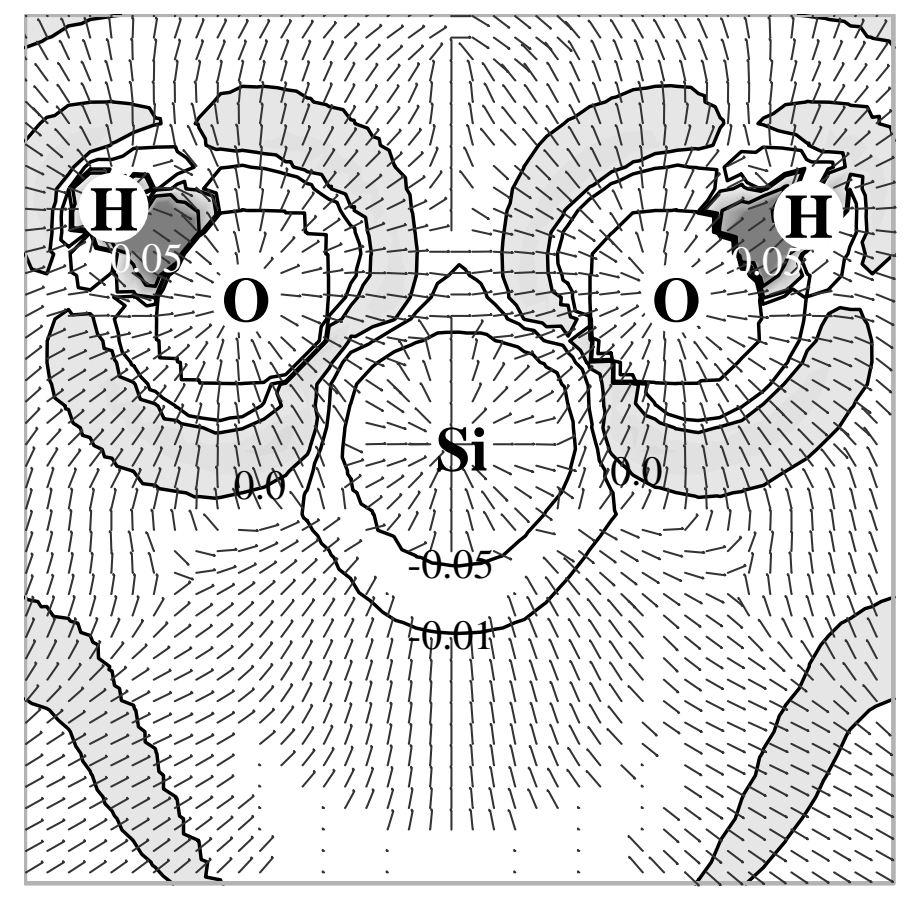

Fig. 3(c) (3/3) JJAP

A. Tachibana 


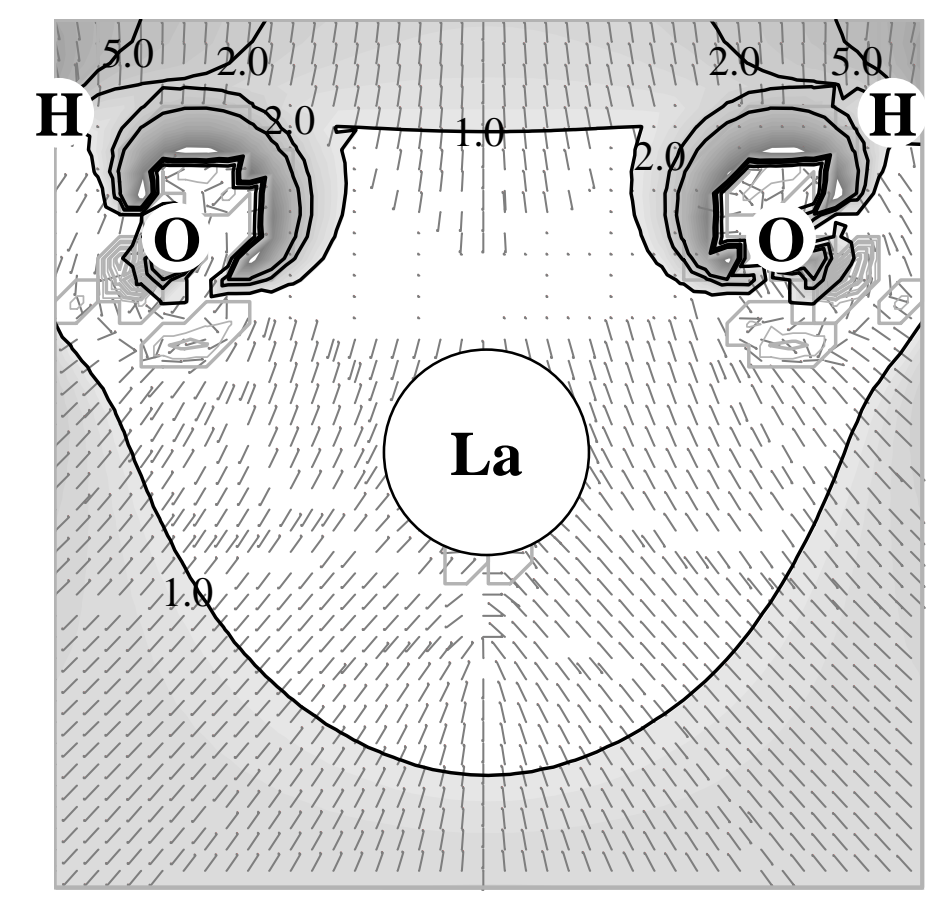

Fig. 4(a) (1/4) JJAP

A. Tachibana 


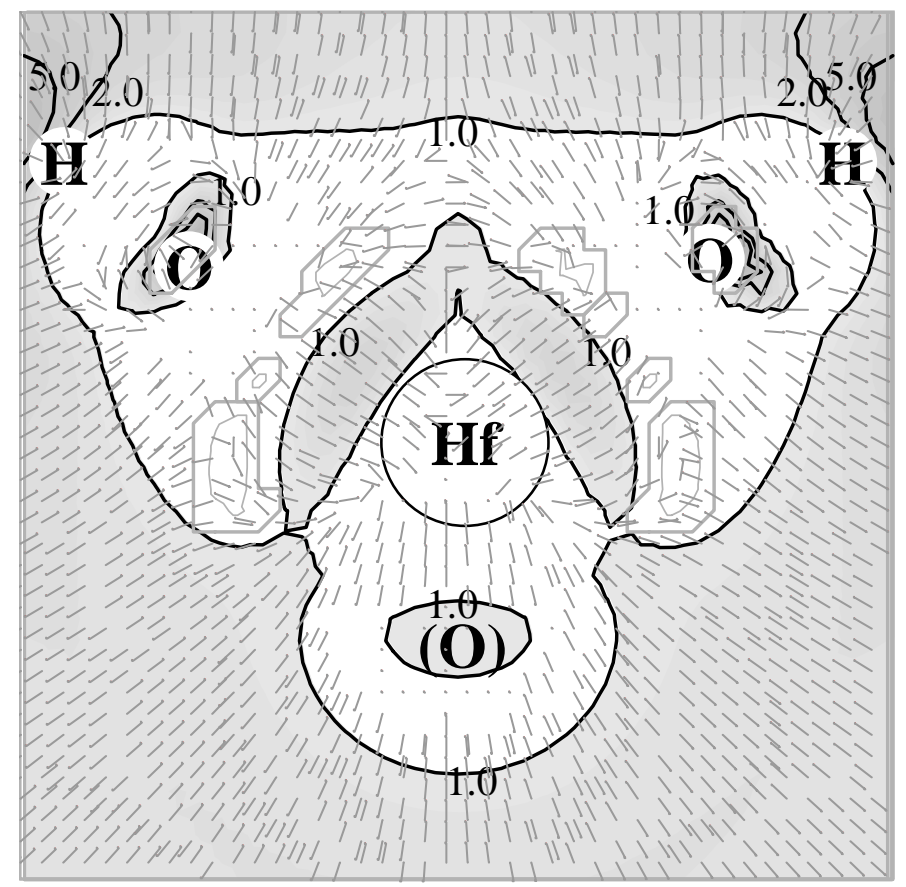

Fig. 4(b) (2/4)

JJAP

A. Tachibana 


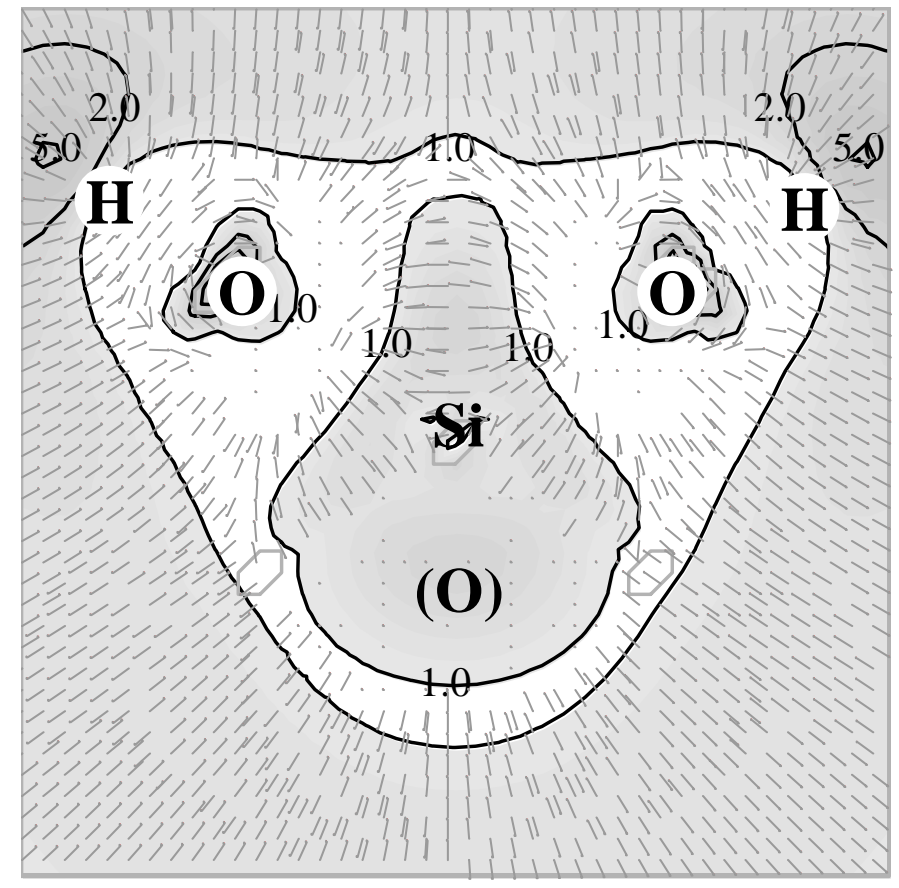

Fig. 4(c) (3/4) JJAP

A. Tachibana 


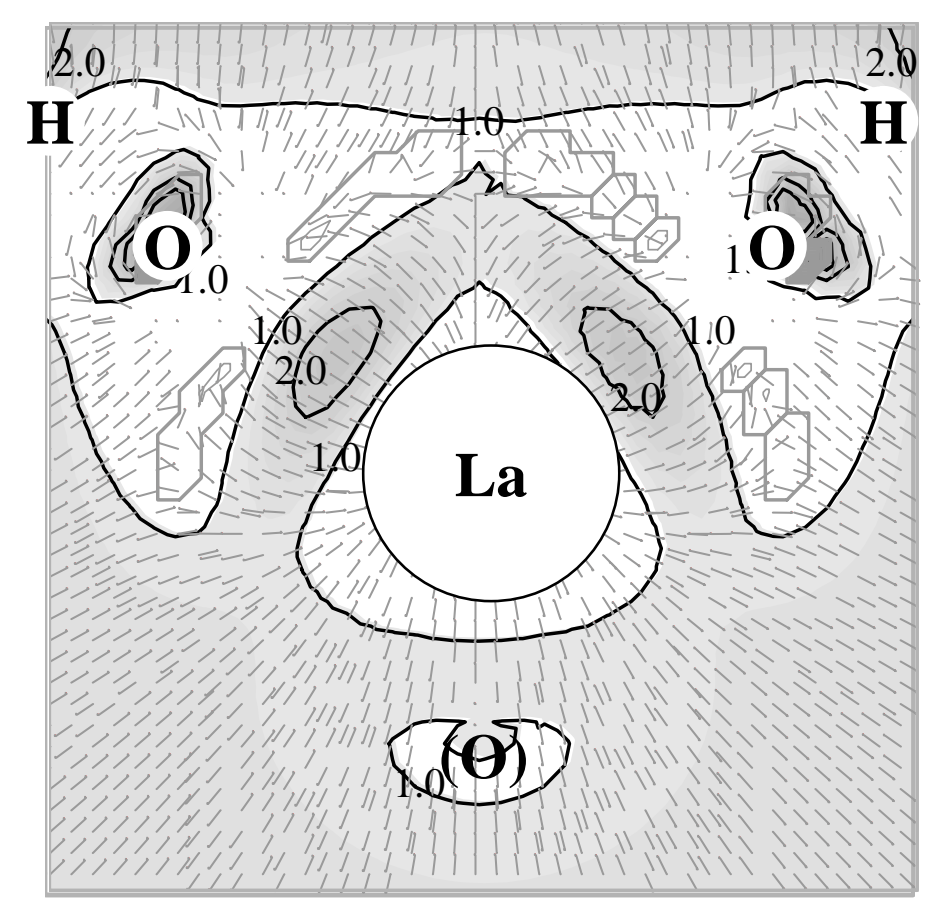

Fig. 4(d) (4/4) JJAP

A. Tachibana 


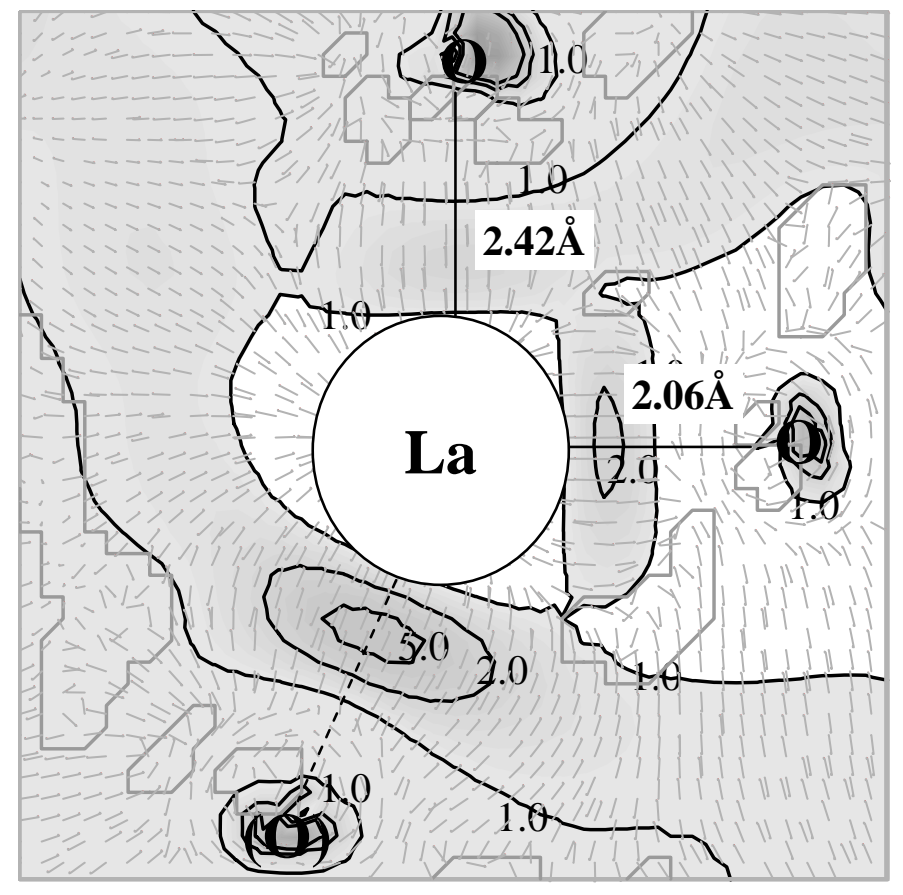

Fig. 5(a) (1/2) JJAP

A. Tachibana 


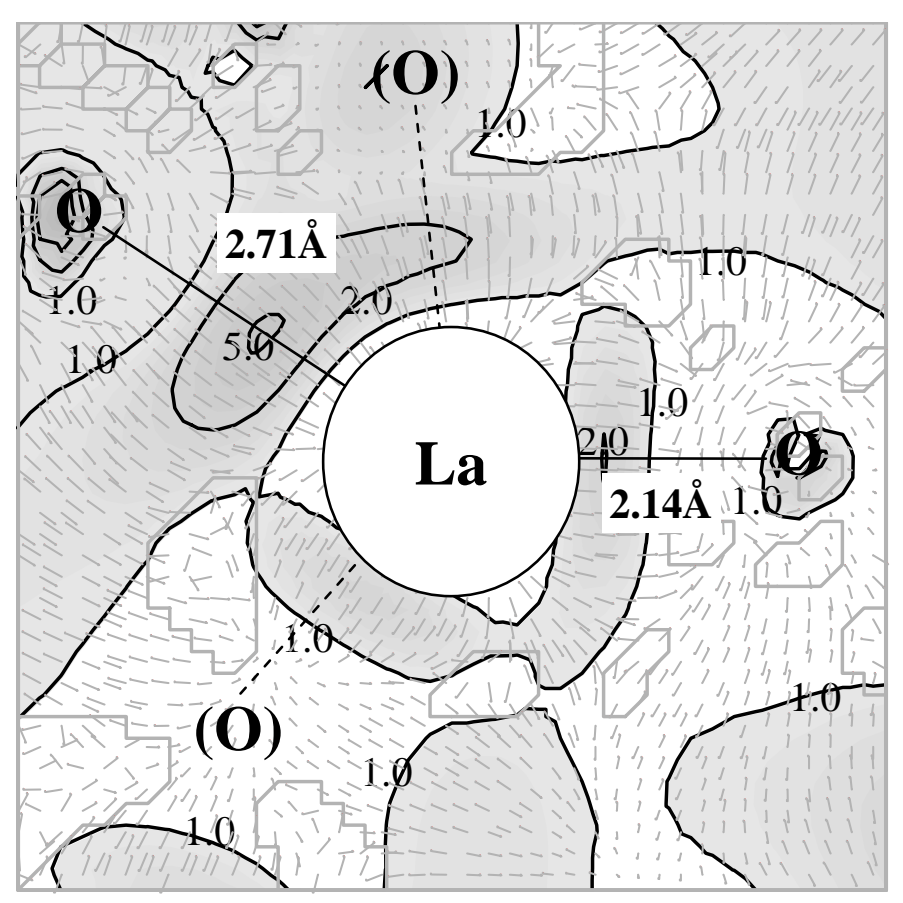

Fig. 5(b) (2/2)

JJAP

A. Tachibana 